\section{Rheumatoid Arthritis and Pri- mary Hyperparathyroidism: Is there a Link?}

\author{
Zulfiqar Abrar-Ahmad \\ Department of Internal Medicine, Geriatrics and Therapeutics, University \\ Hospital of Rouen, 76104 Le Petit Quevilly, France
}

\begin{abstract}
We report a rare case of a concomitant association between primary hyperparathyroidism and rheumatoid arthritis in a 77-yearold female patient. This association between autoimmune disease and non-autoimmune endocrine disorder remains poorly described in the literature and could be fortuitous. Studies should be conducted in both rheumatology and endocrinology in order to gain a better understanding of the possible link.

Keywords: Elderly; Primary hyperparathyroidism; Rheumatoid arthritis
\end{abstract}

\section{Introduction}

In a single patient, multiple auto-immune inflammatory diseases may occur simultaneously. Our report is based on atypical combination of pathologies which has previously seen little description in literature, if at all.

\section{Medical Observation}

A 77 year-old female patient had been admitted to our unit for the treatment of a biological inflammatory syndrome that had been detected in a routine assessment. She was under observation for anxiety and depression, controlled arterial hypertension, right-hand carpal tunnel syndrome and carpal arthrodesis of the left ankle. Routine laboratory tests had been undertaken and had revealed an accelerated initial sedimentation rate of $62 \mathrm{~mm} /$ hour in the first hour (with normal readings being below $30 \mathrm{~mm}$ ). In clinical terms, the patient experienced isolated pains in both shoulders, lasting for 6 months. She complained for several weeks, morning stiffness in both shoulders. The remainder of the examination revealed nothing of note. Tests confirmed the presence of inflammatory disease with a CRP of $103 \mathrm{mg} / \mathrm{L}$ (compared to normal levels below $10 \mathrm{mg} / \mathrm{L}$ ), a sedimentation rate of $82 \mathrm{~mm}$ in the first hour, and high fibrinogen levels of $8.5 \mathrm{~g} / \mathrm{L}$ (normal readings being between 2 and $3.5 \mathrm{~g} / \mathrm{L}$ ).

*Corresponding author: Zulfiqar Abrar-Ahmad, Department of Internal Medicine, Geriatrics and Therapeutics, University Hospital of Rouen, 76104 Le Petit Quevilly, France, Tel: 0627102493; E-mail: abzulfiqar@gmail.com

Citation: Zulfiqar Abrar-Ahmad (2016) Rheumatoid Arthritis and Primary Hyperparathyroidism: Is there a Link. J Orthop Res Physiother 2: 027.

Received: June 10, 2016; Accepted: Aug 10, 2016; Published: Aug 25, 2016
The tests simultaneously revealed moderate hypercalcemia with serum calcium levels of $2.76 \mathrm{mmol} / \mathrm{L}$ (normal values being between 2.2 and $2.6 \mathrm{mmol} / \mathrm{L}$ ), ionized serum calcium levels of $1.45 \mathrm{mmol} / \mathrm{L}$ (normal values being between 1.15 and $1.3 \mathrm{mmol} / \mathrm{L}$ ), corrected serum calcium of $2.88 \mathrm{mmol} / \mathrm{L}$, but no acute renal failure (with creatinine clearance $>90 \mathrm{ml} / \mathrm{min}$ ) and no liver function abnormality. The inflammatory syndrome was tested in the following manner: Tests for infection had proved negative (cyto-bacteriological of urine, aerobic and anaerobic blood cultures, chest X-ray) and a CT scan of the chest; abdomen and pelvic area did not reveal any particular anomalies. The patient, upon questioning, complained of difficulty in articulating the left side of her jaw (with no atherosclerosis on the temporal arteries, without decreased temporal pulsation and ophthalmic symptoms), which led to a biopsy being performed on the temporal arteries, a result of which no histopathological lesions were shown to be present.

Faced with joint pain in both shoulders and a biological inflammatory syndrome, rheumatic tests were initiated. X-rays of the joints of both shoulders revealed only diffuse osteoarthritis, with no signs of erosion. Full immunological testing was requested, with positive results for anti-nuclear antibodies (IFI for Hep-2) at 1/400, with homogenous nuclear fluorescence, an absence of cellular mitosis and negative results for cytoplasmic fluorescence. The Waaler-Rose test was positive at $128 \mathrm{UI}(\mathrm{N}<30)$, and the patient further tested positive for anti-citrullinated peptide antibodies $(52 \mathrm{UI} / \mathrm{ml} ; \mathrm{N}<25 \mathrm{UI} / \mathrm{ml}$ ), indicating the possibility of atypical rheumatoid arthritis. The diagnosis was confirmed after an expert advice from a rheumatologist. The remainder of the immunological tests proved negative. No family history for musculoskeletal diseases.

In parallel, tests for hypercalcemia had been undertaken. Electrophoresis of serum proteins found only an increase in the level of alpha 2 globulins alongside the inflammatory syndrome. Twenty-four hour urinary calcium was slightly elevated, at 339 mg/24h (100-321 mg/24h). Plasma Parathyroid Hormone (PTH) levels has increased slightly, at $71 \mathrm{pg} / \mathrm{ml}$ (with a normal range of $12-65 \mathrm{pg} / \mathrm{ml}$ ) while no vitamin D deficiency was noted (with a rate of $42 \mathrm{ng} / \mathrm{ml}$ ). Morphological examinations (parathyroid scintigraphy) had been undertaken, enabling the detection of a lower right parathyroid adenoma (parathyroid ultrasound was subject to technical limitations due to cervical hyperlordosis). Primary hyperparathyroidism was therefore diagnosed concomitantly with the rheumatoid arthritis.

Full testing was performed in order to determine whether there was a possible link with pheochromocytoma, thereby determining the presence of multiple endocrine neoplasia. Thus, MIBG scintigraphy did not enable the detection of sites of hyper fixation around the two adrenal glands. Moreover, an abdominal MRI did not find any evidence for pheochromocytoma (twenty-four hour metanephrine and urinary normetanephrines could not be interpreted due to the presence of a beta blocker taken for arterial hypertension). We were therefore faced with a 77-year old female patient with a combined potential diagnosis of primary hyperparathyroidism and rheumatoid arthritis. A lower-right hand parathyroidectomy was therefore undertaken in combination with central excision that included two 
reactionary nodes and, on a pathological level, a confirmed non-suspect lower right parathyroid adenoma. This enabled a gradual correction of serum calcium. Moreover, in the context of rheumatoid arthritis, and separately from the surgical intervention, a treatment of corticosteroids was undertaken followed by methotrexate at $15 \mathrm{mg}$ weekly, administered intramuscularly, in combination with folic acid supplements. After several weeks, this resulted in reduced joint pain in both shoulders as well as reduced severity of the inflammatory syndrome. No adverse effects were reported.

\section{Discussion}

The combination of primary hyperparathyroidism with rheumatoid arthritis remains an exceptional one that is very poorly described in the literature to the best of our knowledge. Primary hyperparathyroidism is recognized as a common endocrinopathy and is mainly asymptomatic, except in the case of complications such as kidney stones or fibrocystic osteitis [1]. Its prevalence is estimated at between 1 and 6 in 1,000 [1,2]. Sporadic forms of primary hyperparathyroidism are the most common, but they may occur less frequently in a context of hereditary illness, as in the case of multiple endocrine neoplasia (type 1: in combination with a pituitary adenoma and entero-pancreatic tumors, with the principal gene identified being MEN1; and type 2A, in combination with pheochromocytoma and bone marrow tumor, with RET being the principal identified gene), or in the case of a combination of hyperparathyroidism with mandibular tumors [1-3]. Rheumatoid arthritis is classified as a systematic autoimmune disease, which is to say that the autoimmune response is directed towards antigens that are present in multiple organs).

The genetic environment is inconclusive, with HLADR1 and DR4 genes being present in $30 \%$ and $60 \%$ of cases of rheumatoid arthritis respectively, with immunosenescence that could be caused by premature ageing of the immune system, which affects T-cells in particular. Data seems to show that the immunosenescence observed in rheumatoid arthritis is linked to $\mathrm{DRB} 1^{\star} 04$ [4]. Rheumatoid arthritis is an autoimmune disease that may be linked with other systemic diseases such as systemic erythematosus lupus, Sjogren's syndrome and primary biliary cirrhosis, which may make up a broad umbrella multiple conditions, namely autoimmune syndrome as defined by Humbert et al. [5]. Accordingly, rheumatoid arthritis constitutes a group 2 multiple autoimmune syndromes. The mechanisms behind the physiopathology remain unclear. Recently, it became possible to identify the NALP1 gene, which encodes the NATCH leucine rich repeat, Protein 1 , as a probable factor in vitiligo susceptibility, pernicious anemia (Biermer's disease) and autoimmune thyropathy as well as erythematosus lupus and rheumatoid arthritis. Its role has yet to be determined [6]. This case is a very rare combination of an autoimmune disease, namely rheumatoid arthritis, and a non-autoimmune endocrinopathy, namely primary hyperparathyroidism. Links between primary hyperparathyroidism and monoclonal gammopathy have been described, notably by Clubb et al. in 1964 [7], who were the first to do so. Since then, over ten cases of this kind of link have been reported, although the physiopathology involved remains hypothetical, with the action of monoclonal immunoglobin in the parathyroid tissue, and parathyroid tissue affecting the occurrence of gammopathy (without being required for the condition to persist) having been suggested as possible mechanisms [8]).

Crisp A.J et al., described three cases of association with rheumatoid arthritis and primary hyperparathyroidism. For these authors, this is likely to represent the coincidence of two common diseases but the clinical features are discussed with particular reference to the presence and withdrawal of raised levels of circulating Parathyroid Hormone (PTH). It is argued that raised levels of PTH are potential effects of the rheumatoid disease on bones and joints [9].

\section{Conclusion}

We report about an exceptional case showing a combination of rheumatoid arthritis with primary hyperthyroidism. It is likely that this combination arose fortuitously, but both rheumatological and endocrinological studies should be undertaken in order to gain a better understanding of a possible link.

\section{References}

1. Maruani G, Corniere N, Nicolet L, Baron S, Courbebaisse M, et al (2013) Primary hyperparathyroidism. Rev Med Intern 34: 605-613

2. Melton LJ 3rd (2002) The epidemiology of primary hyperparathyroidism in North America. J Bone Miner Res 17: 12-17.

3. Marx SJ (2011) Hyperparathyroid genes: sequences reveal answers and questions. EndocrPract 17: 18-27.

4. Pillon F, Michiels Y (2013) Epidemiology and physiopathology of rheumatoid arthritis. Actualités Pharmaceutiques 52: 1-2.

5. Humbert P, Dupond JL (1988) Multiple autoimmune syndromes. Ann Med Intern 139: 159-168.

6. Dieude P. (2007) Genetic basis of systemic diseases. Revue du rhumatisme $74: 794-799$.

7. Clubb JS, Posen S, Neale FC (1964) Disappearance of a serum paraprotein after parathyroidectomy. Arch Intern Med 114: 616-620.

8. Dexter RN, Mullinax F, Estep HL, Williams RC Jr (1972) Monoclonal IgG gammopathy and hyperparathyroidism. Ann Intern Med 77: 759-764.

9. Crisp AJ, Helliwell M, Grahame R (1983) The effect of hyperparathyroidism on the course of rheumatoid arthritis. Br J Rheumatol 22: 22-28. 